\title{
COMITÉS DE IMPULSO DE REPARACIÓN COLECTIVA Y SUS DINÁMICAS GRUPALES. CASOS CIÉNAGA DEL OPÓN Y ORGANIZACIÓN FEMENINA POPULAR*
}

\author{
COLLECTIVE REPAIR IMPULSE COMMITTEES AND THEIR GROUP \\ DYNAMICS. CIÉNAGA DEL OPÓN CASES AND POPULAR FEMALE \\ ORGANIZATION
}

\author{
Juan David Pacheco-SÁenz** \\ Claudia QuiJano-MejíA***
}

\section{Resumen}

Objetivo. Comprender las dinámicas grupales de los comités de impulso del corregimiento de Ciénaga del Opón y la Organización Femenina Popular (OFP) y la repercusión de estas en su proceso de reparación, buscando identificar en qué aspectos debe intervenir el profesional del trabajo social. Metodología. Investigación cualitativa, incluyendo la realización de entrevistas semiestructuradas con líderes comunitarios, talleres con integrantes del comité de impulso y observación no participante. Resultados. Se destacan las marcadas diferencias entre un comité de impulso de base comunitaria (Ciénaga del Opón) y uno de base organizativa (OFP) en lo referente a liderazgo, distribución de roles, cohesión del grupo, comunicación entre sus miembros, planeación de actividades y afrontamiento de conflictos. Conclusiones. La intervención del Trabajo Social en comités de impulso de base comunitaria se convierte en un desafío en tanto representan a comunidades fragmentadas por la guerra que hoy deben organizarse para exigir sus derechos ante el Estado.

Palabras clave: comités de impulso, reparación colectiva, dinámicas grupales, trabajo social con grupos.

\begin{abstract}
Objective. To understand the group dynamics of the impulse committees of Ciénaga del Opón and the Popular Females Organization (PFO) and their repercussion in the reparation process, trying to identify the aspects in which the Social Work professional should intervene. Methodology. Qualitative research, including semi-structured interviews with community leaders, workshops with members of the impulse committees and non-participant observation. Results. The great differences between a community-based promotion committee (Ciénaga del Opón) and one based on an organization (OFP)

\footnotetext{
* El artículo es derivado de la investigación Dinámicas organizativas y grupales de los comités de impulso de reparación colectiva en Barrancabermeja, caso de Ciénaga del Opón y la Organización Femenina Popular, desarrollada en el Instituto Universitario de La Paz. Adicionalmente, contó con el apoyo de la Corporación Desarrollo y Paz del Magdalena Medio y la asesoría del Grupo de Investigación en Población Ambiente y Desarrollo, este último en el marco del proyecto de investigación código 110274054663, contrato 451 de 2016, financiado por Colciencias.

*** Instituto Universitario de La Paz. Barrancabermeja, Colombia. E-mail: juandavidpachecosaenz@gmail.com.

(D) orcid.org/0000-0001-5936-5029. Google Scholar

${ }^{* * *}$ Doctoranda en Sociología. Docente Universidad Industrial de Santander. Bucaramanga, Colombia. E-mail cmquijam@uis.edu.co. (D) orcid.org/0000-0002-0224-5621. Google Scholar
} 
are highlighted in terms of leadership, role distribution, group cohesion, communication among its members, planning of activities and confrontation of conflicts. Conclusion. The intervention of Social Work in community based impulse committees becomes a challenge insofar as they represent communities fragmented by the war that today must be organized to demand their rights before the State.

Key words: committees of impulse, collective reparation, group dynamics, social work with groups.

\section{Introducción}

La ley 1448 de 2011 o Ley de víctimas y restitución de tierras estableció los lineamientos para la reparación individual o colectiva a las víctimas del conflicto armado, comprendiendo medidas de restitución, indemnización, rehabilitación, satisfacción y garantías de no repetición. La reparación colectiva constituye un conjunto de medidas que buscan resarcir a aquellos grupos, organizaciones o comunidades que hayan sufrido daños y afectaciones en sus proyectos, prácticas y formas de organización colectivas por ocasión de la guerra. La misma ley establece la conformación de comités de impulso que representen a estas comunidades u organizaciones, con el fin de determinar las medidas de reparación colectiva e interlocutar con las instituciones competentes para materializarlas (Congreso de la República, 2011).

El aumento de casos de reparación colectiva ${ }^{1}$ y, paralelamente, la conformación de comités de impulso convocan a la intervención y fortalecimiento de estos grupos, principalmente cuando provienen de comunidades que han sido desestructuradas por la violencia desplegada por guerrillas, paramilitares y fuerza pública. Muchas veces, debido a su poca experiencia organizativa, los nacientes comités de impulso no tienen un objetivo claro para el desarrollo de su gestión, cuentan con poca formación referente al manejo de procesos comunitarios y presentan dificultades en relación al liderazgo, al trabajo en equipo, los roles, el manejo de conflictos, la cohesión interna, la comunicación, entre otros. Por el contrario, los comités de impulso que representan a organizaciones sociales cuentan con la experiencia necesaria para lograr mayores niveles de gestión y concertación con las entidades competentes de implementar las medidas de reparación establecidas.

Los casos de la comunidad del corregimiento Ciénaga del Opón y la Organización Femenina Popular (OFP), en el municipio de Barrancabermeja, representan dos situaciones disímiles en la conformación y desarrollo de los comités de impulso. Ambas colectividades enfrentaron actos de violencia por parte de los grupos armados e iniciaron el proceso de reparación contemplado en la ley 1448 de 2011. Sin embargo, los logros alcanzados por cada comité de

\footnotetext{
${ }^{1}$ La Coordinación Nacional de Sujetos de Reparación Colectiva menciona para mayo de 2018, 615 casos reconocidos mediante resolución que aún no han sido reparados (Mesa Nacional Coordinación Sujetos de Reparación Colectiva, 2018).
} 
impulso evidenciaron las diferencias entre el proceso de reparación de una comunidad y de una organización social. Situación que podría entenderse, en parte, a las dinámicas grupales desarrolladas por cada uno de los comités de impulso.

En otras palabras, con la conformación de los comités de impulso de reparación colectiva se inicia un proceso grupal que amerita ser entendido. Según Schafer (1984) un grupo es un:

\begin{abstract}
Número de miembros quienes, para alcanzar un objetivo común (objetivo de grupo), se inscriben durante un período de tiempo prolongado en un proceso relativamente continuo de comunicación e interacción y desarrollan un sentimiento de solidaridad (sentimiento de nosotros). Son necesarios un sistema de normas comunes y una distribución de tareas según una diferenciación de roles específica de cada grupo. (pp. 26-27)
\end{abstract}

Para la consecución de sus objetivos, los miembros del grupo desarrollan un proceso de interacción constante que genera una estructura de interdependencia, es decir, una dinámica grupal. Estas relaciones de interacción cuentan con una serie de propiedades que se enmarca en un patrón de dirección o liderazgo, en las funciones o roles que se ejercen dentro del grupo y que se relaciona con el nivel de cohesión o armonía del mismo, siendo la comunicación trasversal a dicha dinámica y la planeación de los procesos base de la estructura organizacional (Knowles y Knowles, 1962).

El liderazgo es un proceso grupal en el que están implicados el líder, los seguidores y la situación en la que se ejerce; es la capacidad de influir a los otros miembros del grupo para la consecución de la meta u objetivo grupal (Morelo, 2012). El rol se encuentra en correlación con el estatus del miembro del grupo y hace referencia al comportamiento de una persona que ocupa determinada posición (estatus) en el grupo, implicando una serie de derechos y deberes (Huici, 2012). La cohesión se entiende como todos aquellos elementos que permiten mantener a los miembros del grupo unidos, se destacan el contacto, la existencia de un "nosotros" entre sus miembros y la interdependencia entre ellos para la consecución de los objetivos individuales y grupales (Roda, 1999).

Por otra parte, la planeación como elemento importante para la consecución de los objetivos del grupo, se entiende como,

Un proceso de preparar un conjunto de decisiones para la acción futura, dirigida al logro de objetivos por medios preferibles. Es un proceso, una actividad continua que no termina con la formulación de un plan, sino que implica reajuste permanente entre medio y fines. (Ander Egg, 2007, p. 25) 
El análisis de la dinámica grupal también comprende la comunicación y la forma como se resuelven los conflictos dentro del grupo. De acuerdo con Gouran et al. (como se citó en Macías, 2003), la comunicación en el grupo debe entenderse como un instrumento que busca lograr el consenso para la toma de decisiones y la resolución de los problemas. Por su parte, los conflictos son situaciones que hacen parte de las relaciones humanas y la cotidianidad. Algunos de ellos pueden desencadenar hechos de violencia, ya sea directa, que se manifiesta física o psicológicamente; estructural, dada por factores sociales que producen hechos de desigualdad y exclusión, causado por un sistema político, económico o social excluyente y, por último, la violencia cultural dada por la estigmatización hacia otros por creencias o prejuicios (Galtung, 1999). De ahí la importancia de una adecuada tramitación que permita más que su solución, su transformación.

Desde la profesión-disciplina del trabajo social la comprensión de estas dinámicas grupales permitirá mejorar la intervención en estos contextos, entendiendo que los procesos de intervención con los comités de impulso de reparación colectiva constituyen lo que se denomina trabajo social con grupos que, en palabras de Ander Egg, está orientado "al crecimiento de los individuos en el grupo y a través del grupo y el desarrollo del grupo hacia tareas específicas y como medio para actuar en ámbitos sociales más amplios” (Ander Egg, 2003, pp. 137, 138).

Los comités de impulso son el tipo de grupo que tiene por objetivo cumplir una labor, una función, una responsabilidad que les ha sido asignada por la comunidad u organización que representan. Y aunque no se orienta al crecimiento personal, la sola participación en el grupo contribuye al desarrollo de sus integrantes al potenciar habilidades como el liderazgo, la toma de decisiones, el trabajo en equipo, la resolución de conflictos, entre otros (Gnecco, 2005). En esa medida, "el trabajador social se sitúa "al lado" del grupo e intenta fomentar su capacidad para auto-organizarse y ser efectivo en el logro de sus objetivos sociales, al mismo tiempo que intenta potenciar líderes que lo dirijan” (Rossell Poch, 1998, p. 112).

Para el caso, la actuación profesional con y en los comités de impulso de reparación colectiva exige a los profesionales del trabajo social conocer la dinámica grupal de los comités, pues de este modo es posible potencializar la capacidad del grupo para satisfacer las necesidades de sus participantes y alcanzar sus objetivos de reparación. En otras palabras, conocer la dinámica grupal de los comités de impulso es un paso necesario para, desde la intervención profesional, promover el empoderamiento de los comités de impulso para que lideren y representen a sus comunidades en el proceso de reparación colectiva.

En concordancia con lo expuesto, el presente artículo busca comprender las dinámicas grupales de los comités de impulso del corregimiento de Ciénaga del Opón y la OFP de Barrancabermeja y la repercusión que estas tuvieron en su proceso de reparación colectiva. Concomitantemente, se busca identificar en qué aspectos puede intervenir el trabajador social 
para posibilitar que estos grupos logren sus objetivos en el marco de la reparación colectiva. Para ello, la investigación adoptó un diseño cualitativo que incluyó técnicas de recolección de información como entrevistas semiestructuradas a los representantes de cada comité de impulso, tres talleres con la participación de los integrantes de cada comité y la observación participante que se realizó durante un año de trabajo de campo desarrollado entre el segundo semestre de 2017 y el primer semestre de 2018.

\section{Discusión}

\section{La comunidad de Ciénaga del Opón y la Organización Femenina Popular: procesos de reparación colectiva y conformación de comités de impulso}

El corregimiento de Ciénaga del Opón se ubica en Barrancabermeja (Santander), está compuesto por ocho veredas y cuenta con una población aproximada de 1250 habitantes, distribuidas en 267 familias. Los primeros hechos de violencia vividos por la comunidad de Ciénaga del Opón se relacionan con la presencia y control territorial de las FARC-EP en la región, principalmente los frentes 12, 13 y 24. Así, el 8 de febrero de 1986 esta guerrilla realizó una incursión armada al corregimiento que ocasionó la muerte del inspector de policía y el primer desplazamiento a la cabecera municipal. Al final de la década de 1990, la expansión nacional del paramilitarismo y la disputa territorial con las guerrillas tuvo como resultado el ingreso de las Autodefensas Campesinas del Magdalena Medio al corregimiento el 28 de septiembre de 1998 y el consecuente desplazamiento de 70 familias. La situación se agudiza cuando los paramilitares quemaron la oficina de Telecom, el puesto de salud y saquearon la cooperativa comunitaria de Cootresbocas, además de varios asesinatos y desapariciones, que provocaron el desplazamiento masivo el 2 de marzo del 2000 de los pobladores de las veredas Cabecera, Candelaria, Florida y Neques hacia el casco urbano de Barrancabermeja, en donde se asentaron en el Colegio Normal Superior Cristo Rey por un periodo de dos años (PDPMM, 2012).

Por su parte, la Organización Femenina Popular es una organización defensora de los Derechos Humanos integrales que nace en 1972 en Barrancabermeja con el apoyo de la iglesia católica, pero que desde 1988 inicia un proceso independiente que se consolida en la ciudad y hacia 1995 se expande hacia otros municipios del Magdalena Medio. La OFP centra su accionar en los principios de la civilidad y la autonomía, resistiendo a toda tipo de violencia (OFP, s.f). A partir de 1998 con el recrudecimiento del conflicto armado en la ciudad, producto de las incursiones paramilitares y la disputa territorial con las guerrillas, la OFP con su postura de hacer frente a toda forma de violencia se convirtió en blanco de ataques sistemáticos por parte de los grupos paramilitares, reportando alrededor de 140 crímenes cometidos en contra de la organización y sus miembros, entre ellos se registran asesinatos, desapariciones, amenazas y hostigamientos contra integrantes de la OFP y sus familias, así como el ataque y saqueo a las sedes de organización (Obando, 2016). 
El proceso de la reparación colectiva se desarrolla a través de una serie de pasos, inicia con el reconocimiento del sujeto colectivo por parte de la Unidad para la Atención y Reparación Integral a las Víctimas (UARIV), el diagnóstico del daño, la construcción de medidas de reparación y finaliza con la implementación y seguimiento a las mismas. Cada paso es acompañado por los comités de impulso previamente conformados y elegidos por los sujetos colectivos, en este caso, por la comunidad de Ciénaga del Opón y la Organización Femenina Popular entre 2012 y 2013, cuando iniciaron el trámite de su reparación colectiva a través de la modalidad "por oferta", es decir, la UARIV le ofreció a la comunidad y a la organización ser parte del proceso ${ }^{2}$.

Para la conformación del comité de impulso de Ciénaga del Opón se delegaron 15 miembros de las veredas de Cabecera, Neque, Florida y Colorada (aunque el corregimiento cuenta con ocho veredas) y para el caso de la OFP se conformó un comité de víctimas con participantes de los municipios de San Pablo, Sabana de Torres, Yondó, Girón y Barrancabermeja, con un total de 30 mujeres aproximadamente.

\section{Dinámicas grupales de los comités de impulso de reparación colectiva}

En la consecución de sus objetivos, los grupos desarrollan procesos de interacción constante entre sus integrantes, configurando una dinámica grupal propia que incluye: el liderazgo, la distribución de los roles, la cohesión del grupo, el tipo de comunicación que se establece entre sus miembros, la planeación para la consecución de sus objetivos y la forma de afrontar los conflictos. Por ello, para entender el proceso grupal de los comités de impulso de Ciénaga del Opón y la OFP es necesario conocer en detalle cómo se expresan estos elementos al interior de cada grupo.

El liderazgo. La comprensión de esta categoría implica la contextualización de la experiencia de ser líder. Para el caso del comité de impulso de Ciénaga del Opón, conformado principalmente por personas dedicadas a labores como la pesca y la agricultura familiar, hasta ese entonces el ejercicio del liderazgo de sus habitantes se había desarrollado en espacios como las juntas de acción comunal (JAC) y las juntas administradoras locales (JAL), que posibilitaban la interlocución con los gobiernos municipales y la participación en escenarios como la elaboración del presupuesto participativo, además de intermediar en problemas cotidianos en sus comunidades. Es la coyuntura del conflicto armado y, en específico, la convocatoria de la UARIV para participar en el proceso de reparación colectiva lo que llevó a replantear de forma imprevista el ejercicio del liderazgo en un nuevo espacio. "De pronto por la confianza que me tienen los compañeros de lucha y de pronto esa confianza me la he ganado a través de

\footnotetext{
${ }_{2}^{2}$ También existe la modalidad por "demanda", efectuada cuando la comunidad solicita a la UARIV el ingreso al proceso de reparación colectiva.
} 
mi respeto y sinceridad con ellos" (Líder Ciénaga del Opón, comunicación personal, 10 de octubre de 2017).

Así, para la comunidad la reparación colectiva se convierte en un reto que se inicia con la conformación del comité que impulsaría el proceso. La UARIV convoca a los habitantes de las veredas sujetos de reparación para delegar a las personas que liderarían el proceso a través del comité de impulso, siendo elegidos por la comunidad personas que se destacaban por su capacidad para hablar en público, conocimiento y desempeño frente al tema de reparación, capacidad de gestión, entre otros. En concordancia, puede afirmarse a partir de los postulados de la teoría de los rasgos de Ralph Stodgill (como se citó en Yukl, 2008), que si bien estas características son importantes para la selección de las personas que se desempeñan como líderes, por sí solas no garantizan el éxito; es fundamental que estos rasgos tengan correspondencia con las características, las actividades y las metas de los otros integrantes del grupo. En otras palabras, esta teoría apunta a determinar qué tipo de persona debe ejercer la labor de liderar, pero no garantiza el éxito de lo emprendido (Yukl, 2008).

Para el caso del comité de impulso de la Organización Femenina Popular, su esquema de liderazgo va en sincronía con la misma estructura de la organización. Es de recordar que la OFP nace como defensora de derechos humanos y del empoderamiento social y político de las mujeres del Magdalena Medio y lleva cerca de 47 años en esta labor. La experiencia acumulada en esta larga trayectoria organizativa y los relevos generacionales dentro de la organización han permitido el sostenimiento de los ideales y la formación y crecimiento personal de mujeres que hoy se proyectan como líderes comprometidas tanto con el comité de impulso como con la OFP. "Hemos tenido muchos espacios, inclusive hemos tenido escuelas donde las líderes van y hacen su proceso de un año, dos años. Hay muchos talleres que han sido asesorados por ONGs a nivel nacional y a nivel internacional” (Líder OFP, comunicación personal, 29 de octubre de 2017).

En la experiencia de la OFP pueden ser identificados los componentes del denominado liderazgo transformacional (Bass \& Riggio, 2006): 1). La influencia e inspiración de las lideresas basadas muchas veces en el carisma que estas proyectan y acompañadas de la capacidad de resistencia; 2). La estimulación intelectual de las afiliadas, quienes participan en procesos de formación, estimulación de la creatividad e innovación sin críticas a errores personales; 3). El tratamiento individualizado de las integrantes, que permite identificar a aquellas que requieren más apoyo para el cumplimiento de las distintas tareas.

Los procesos de liderazgo en el comité de impulso de la OFP no se reducen a la coyuntura que ofrece la reparación colectiva, por el contrario, es un proceso que viene desarrollándose con anterioridad, por cerca de 45 años y que ha permitido lograr la incidencia política necesaria para la implementación del proceso reparador. Caso contrario sucede con el comité de 
impulso de Ciénaga del Opón, cuyo proceso de liderazgo en el marco de la reparación ha carecido de esta experiencia previa que facilite la incidencia política, generando desventaja a la hora de interlocutar con las entidades concernientes. Dicha situación también se atribuye a que el comité de impulso surge solo por y para el proceso de reparación, años después de haber vivido un hecho violento que obligó a los habitantes del corregimiento a radicarse en Barrancabermeja. Por ello, deben establecerse procesos orientados no solo a la formación de sus integrantes, sino a la recuperación del tejido social y a la construcción de un proyecto colectivo comunitario.

Roles y funciones. Los roles en el grupo pueden ser formales, es decir, son establecidos por los grupos de acuerdo con las actividades y metas a cumplir o, por el contrario, roles informales que van surgiendo con el trasegar de la interacción grupal, siendo este último el más notorio en pequeños grupos.

Para el caso de Ciénaga del Opón, los integrantes desde el segundo semestre del 2016 han estipulado una serie de roles o funciones específicas a cada uno de sus miembros de acuerdo con sus capacidades, disposición y rasgos (estatus). Dichos roles se encuentran distribuidos de la siguiente manera: vocero, moderador, relator, enlace y personal de apoyo. El comité de impulso de Ciénaga del Opón en sus inicios (2013) había establecido roles de tipo informal caracterizados por su orientación a la tarea y, en la medida que el proceso de reparación se iba complejizando (negociaciones con la administración municipal, UARIV, discusiones de avances de las medidas, etc.), tuvieron que establecer funciones que potenciaran su dinámica.

\footnotetext{
Tenemos distintas habilidades y saberes pero nos podemos complementar y apoyarnos.

En el comité estamos débiles y cada uno debe darle la mano al otro para salir adelante.

Todos tenemos un rol importante así no lo parezca, creo que debemos potenciarlo con la ayuda del otro. (Líderes Ciénaga del Opón, comunicación personal, 7 de diciembre de 2017)
}

Sin embargo, la asignación de estos roles no ha sido un elemento determinante tanto en la dinámica grupal como en los resultados del proceso de reparación, ya que si se analiza cada función de los integrantes se evidencia el no cumplimiento de las labores asignadas. Por ejemplo, se tiene un relator pero no hay evidencias referentes a la gestión documental del comité de impulso, existe un moderador de las reuniones pero no se fijan reglas o parámetros en dichos encuentros, existe un vocero para que exprese lo acordado por el comité pero no existen encuentros previos para tomar las decisiones, entre otros aspectos. 
La OFP como organización maneja dentro de su estructura un esquema de tipo circular, que cuenta con un equipo de dirección encargado de los temas nacionales, entre estos la reparación colectiva, con el apoyo de diferentes equipos de coordinadores, comité de víctimas y equipos de apoyo ampliados. El comité de impulso de la OFP está compuesto por alrededor de 30 mujeres que distribuyen su accionar territorialmente en los diferentes municipios donde hace presencia la organización. Cabe recordar que para la OFP la figura de comité de impulso es el mismo comité de víctimas. Aunque la OFP maneja un sistema organizativo estructurado, su comité de víctimas o de impulso, se compone de una estructura sencilla, donde se estipulan roles de coordinación y roles de apoyo.

Se deduce entonces que el comité de impulso de la OFP define una serie de roles informales, donde las funciones de las coordinadoras están centradas en socializar a las bases lo referente a la reparación colectiva y otros procesos que adelante la coordinación nacional, así como acompañar las actividades respectivas del proceso. Por ello, se determina que gran parte de los resultados del proceso reparador dependen más de la coordinación nacional que del mismo comité de impulso, ya que este opera en los territorios según las directrices nacionales y los roles no solo se orientan a la tarea o al cumplimiento de objetivos, sino a mantener la armonía y sostenimiento de las bases en cada sector.

Cohesión. La definición de cohesión en el marco de las dinámicas grupales se entiende como todas aquellas acciones que permiten mantener a sus miembros unidos, en otras palabras, es el campo total de fuerzas que actúan sobre los miembros de un grupo para permanecer en él (Festinger, Schachter y Back, 1950). Para determinar la cohesión de los comités de impulso de reparación colectiva de Ciénaga del Opón y la OFP, se tuvo en cuenta la percepción individual de algunos de sus integrantes sobre el comité y cómo esta puede influenciar en los resultados del proceso de la reparación colectiva.

\footnotetext{
Porque a través de todas las circunstancias, de todos los talleres, hemos ya tenido una posición, yo por lo menos me siento en la quinta, porque nosotras ya estamos agrupadas, más solidarias, estamos con un solo pensamiento. Estamos todos juntas compartiendo. (Líder OFP, comunicación personal, 7 de diciembre de 2017)
}

Dentro de los testimonios recogidos se percibe que el comité de impulso de la OFP muestra un balance favorable, evidenciando elementos de atracción interpersonal entre los miembros del grupo (compañerismo, amistad, camaradería, etc.) y a su vez una atracción hacia las actividades y objetivos del grupo, como lo señala Shaw (1983). Aunque en la actualidad manifiestan inconformismo por el avance del Plan Integral de Reparación Colectiva (PIRC) -son 41 medidas de las cuales nueve están completas, nueve están parcialmente completas y 23 por completar-, la fuerte cohesión del comité de impulso de la OFP es reflejo de la dinámica 
propia de la organización, afianzada desde el punto más álgido del conflicto, hasta el inicio del proceso de reparación colectiva. Esto ha implicado no sólo el sostenimiento y el aumento de las afiliadas a la organización, sino el fortalecimiento de los equipos de apoyo del comité de víctimas o impulso, lo cual ha generado un respaldo a las gestiones de la coordinación nacional, sumado al empoderamiento que ofrece el comité de la OFP frente a los temas de género, emprendimiento y reparación colectiva a sus miembros.

Por su parte, la cohesión dentro del comité de impulso de Ciénaga del Opón está determinada por la relación del comité con otros actores. A pesar de que esas relaciones muchas veces parecieran dejar un balance negativo, esta situación no disminuye la cohesión sino que la fortalece.

En la etapa cuatro, creo que se han vencido muchas dificultades y debemos seguir trabajando para fortalecer la confianza al interior del comité. (Líder Ciénaga del Opón, comunicación personal, 7 de diciembre de 2017)

Según Hogg (1992), estos hechos aparentemente contradictorios se originan por la necesidad de identificación social y auto categorización, el identificarse no sólo como individuos, sino como parte de un grupo con el que comparte rasgos, valores o características similares que los diferencia de otros grupos y que permite ejercer comportamientos de acuerdo con pautas normativas establecidas por ellos mismos. Es decir, los factores externos al comité que se relacionan con el proceso reparador (acercamientos con la UARIV, discusiones con la administración municipal frente a los planes de acción territorial, reuniones con la mesa de víctimas, intervenciones ante entes de control) hacen que aquellas aparentes diferencias interpersonales pierdan importancia y, contradictoriamente, se fortalece la unidad grupal por la necesidad de establecer criterios colectivos frente a los procesos de negociación con el Estado y sus instituciones.

El abordaje de los conflictos. Con la conformación de los comités de impulso, que surgen como grupos para gestionar las medidas adoptadas en el proceso de reparación, también nace el desafío de resolver los conflictos que emergen en este nuevo grupo, lo que implica que los miembros del comité sienten posiciones, expliciten sus intereses particulares y manifiesten sus opiniones y divergencias, de manera respetuosa y con el propósito de resolver las diferencias.

En ambos comités se evidencia la existencia de conflictos dados por las contradicciones entre los distintos intereses de los integrantes del comité; además, de las actitudes relacionadas con la disposición y grado de apertura para abordar los conflictos y los comportamientos con que se afrontan estos mismos. En los comités de impulso se refrenda el conflicto como una constante de las dinámicas grupales. Sin embargo, es importante resaltar que los integrantes 
de los comités buscan mantener las buenas relaciones mediante el establecimiento de normas y acuerdos, acordes al estilo e historia de cada comité.

Lederach (1989) identifica cinco estilos de afrontamiento de los conflictos: (i) el estilo competir que hace referencia al ganar el todo por el todo, se busca imponer el criterio personal sobre los demás; (ii) el estilo acomodar tiende a ser lo contrario al anterior y busca ceder con el fin de mantener buenas relaciones, renunciando a sus intereses personales; (iii) el estilo evitar que busca eludir el conflicto por considerarse en desventaja o no ser el momento apropiado; (iv) el estilo cooperar que propende por los intereses de todas las partes, siendo un estilo adecuado cuando se quiere mantener las buenas relaciones; y finalmente, (v) el estilo negociar que busca llegar a un punto intermedio en el que cada una las partes ha cedido un poco.

En cada uno de los comités se indagó sobre el estilo de resolución de conflictos que predomina, dejando claro que todos los estilos son útiles según las circunstancias. Para ello, se realizó el test ¿Cómo abordamos los conflictos? (Fernández, Cancino, Botero y Prada, 2016), que retoma los postulados de Lederach (1989) sobre el tema y está compuesto por veinte refranes que deben ser escogidos por los participantes, ya sea por su afinidad con el refrán o porque lo aplican a su diario vivir, para determinar el estilo cómo se abordan los conflictos.

Como resultado se obtuvo que en el comité de impulso de la OFP sobresalen los estilos cooperar y evitar para resolver los conflictos dentro del comité, buscando mantener las buenas relaciones y conservar la armonía entre las mujeres que lo integran. Frente al estilo que se considera adecuado para abordar los conflictos que surjan con las instituciones correspondientes en el proceso de reparación colectiva, manifestaron que el estilo competir ha prevalecido en las distintas gestiones.

\begin{abstract}
Nos toca tener posturas de competir, la OFP es uno de los que ha avanzado en los proceso de reparación y nos toca seguir exigiendo, nos toca a veces hacer la tarea de ellos (Estado), de investigar. Así, con el estancamiento que hay con la unidad de víctimas, hemos ido trabajando para sacar adelante, nos toca seguir guerreando como tiburón. (Líder OFP, comunicación personal, 7 de diciembre de 2017)
\end{abstract}

Dentro del comité de impulso de Ciénaga del Opón también prevalecen los estilos cooperar y evitar, siendo más frecuente este último, así, los conflictos en comité pueden ser más persistentes, ya que no se han implementado herramientas claras para solucionarlos/ transformarlos.

Las dificultades que más se presentan en el comité, diría yo y voy a decirlo claro, mucho egoísmo, muchos líos personales "que yo lo que quiero es plata” pero no pasa de ahí. Nosotros al interior del comité 
como hemos hecho siempre le llamamos la atención a los compañeros que están en desacuerdo. (Líder Ciénaga del Opón, comunicación personal, 7 de diciembre de 2017)

Si bien en muchos casos es importante evitar los conflictos, resultaría necesario establecer criterios grupales donde el estilo de cooperación sea el primer lineamiento en la consolidación del trabajo en equipo. Adicionalmente, los comités coinciden en que frente al proceso de reparación se requiere o se han asumido posturas de "competir" ante las entidades correspondientes, dado el no cumplimiento de las medidas de reparación colectiva.

Formas de comunicación de los comités de impulso. Si bien cada uno de los elementos de las dinámicas grupales de los comités de impulso se han descrito separadamente, es de aclarar que todas ellas están relacionadas entre sí; no se puede hablar de liderazgos sin haber roles definidos, el manejo de los conflictos está estrechamente relacionado con la cohesión y, equivalentemente, la comunicación es transversal a toda la dinámica grupal de los comités de impulso, siendo un factor determinante en el desarrollo de la gestión de la reparación colectiva.

La comunicación debe ser entendida no solo como un proceso de transmisión de información, sino como un "proceso social” que establece consentimientos mutuos (toma de decisiones), que orienta una cultura grupal en la que se pueda establecer liderazgos, las formas de negociación, entre otros aspectos (Macías, 2003). En este sentido, se hizo un acercamiento para describir el tipo de comunicación que prevalecía en los comités de impulso a partir de la observación de la toma de decisiones y cómo estas trascienden los grupos o comunidades que representan.

En el caso del comité de impulso de Ciénaga del Opón, la comunicación en su interior es de tipo informal, es decir, no se establecen criterios oficiales o estructurados, surge de forma espontánea y verbal, siendo posible que los integrantes se comuniquen directamente entre sí y con los demás miembros de la comunidad. Sus parámetros para el establecimiento de acuerdos, normas y decisiones se estipulan bajo consenso.

Las decisiones, si alguno de los compañeros hace una propuesta y la vemos de común acuerdo, todos llegamos a un consenso y ahí tomamos la decisión. Para socializar a la comunidad, en algunas ocasiones, y en otras no, por la sencilla razón de que muchas decisiones se han tomado y es que ¡se tiene que tomar ya! porque estamos aquí en Barranca y no podemos y no se pueden dejar las cosas pues toca asumir esa responsabilidad. (Líder Ciénaga del Opón, comunicación personal, 10 de octubre de 2017) 
En lo referente a la comunicación y retroalimentación con la comunidad en general en los aspectos de la reparación colectiva, se evidencia que algunos habitantes del corregimiento no ven favorable la gestión del comité de impulso, por considerar que la gestión que se realiza es para beneficio particular. Por consiguiente, se puede entender que el comité deberá emplear acciones comunicativas que permitan mayor respaldo comunitario a través de posibles canales como las juntas de acción comunal.

Frente a este tipo de comunicación informal que utiliza el comité de Ciénaga del Opón, se puede señalar como punto a favor que permite en cierta manera procesos ágiles de interacción interna, sin la necesidad de grandes procesos estructurados para la circulación de la información. A su vez, en el intercambio de información con las entidades responsables del proceso de reparación colectiva se ha avanzado en el reconocimiento del comité como receptor y canal de la gestión, pues anteriormente las entidades realizaban contactos con algunas JAC, desconociendo el papel articulador que desempeña el comité de impulso.

Es importante aclarar que aunque el estudio se centró en los comités de impulso, para el caso de la OFP su dinámica está ligada a la estructura grupal de la organización como un todo, lo que implica un esquema comunicativo formal donde los mensajes se encuentran oficializados o establecidos, ya sea por un orden jerárquico o normativo de la organización y, de ese modo, la información fluye de forma circular entre los distintos espacios de la organización que comprenden un número significativo de miembros en cada uno de ellos (asamblea, equipo de dirección, equipo coordinador, comité de víctimas, equipo de apoyo, entre otros).

Dentro del comité de impulso de la OFP (o comité de víctimas, como es llamado en la organización), los mensajes de la organización son dados por la respectiva coordinadora del comité zonal o territorial, en concordancia con los principios y políticas de la OFP, asimismo, estos mensajes son repasados por la coordinadora a los otros equipos de la organización. De este intercambio de información se deja evidencia escrita como actas, informes, relatorías y sistematizaciones. Este esquema formal resulta ventajoso ya que la información llega sin distorsiones a las bases del comité y de la misma organización, lo que no solo implica conocimiento de los aspectos de la reparación, sino también procesos de empoderamiento entre las afiliadas.

El flujo de información entre los comités de impulso y los sujetos que representan se da de manera opuesta en cada una de las experiencias analizadas: desde la OFP la información se canaliza a través de sus máximos órganos y baja hasta el comité de víctimas, y para el caso de Ciénaga del Opón, la información, primero, llega a su comité de impulso y posterior a las veredas del corregimiento. 
La planeación. Si bien el proceso de reparación colectiva obedece a una planeación de orden nacional que busca ser operada a través de las distintas instituciones en los territorios donde se encuentran los sujetos colectivos, existe un margen de planeación por parte de los comités de impulso en lo referente a las estrategias y acciones a desarrollar en cada etapa del proceso local.

Sin embargo, en el comité de impulso de Ciénaga del Opón se ha afrontado el proceso de reparación a partir de las directrices de las instituciones, del día a día y el ritmo que estas imponen, siendo la planeación una inexistente herramienta de formulación de acciones y estrategias en este comité, generando que se impongan la perspectiva de terceros al momento de determinar las acciones del comité. Entonces, se hace imperativo que, desde el sujeto de reparación en cabeza de su comité, se generen posibles acciones y alternativas para el seguimiento del cumplimiento de las medidas de reparación.

Situación contraria se evidencia en el caso de la OFP, pues hubo un diseño previo de las acciones que les permitiría abordar la reparación colectiva en sus distintas etapas. Desde la OFP se planeó la realización de tres talleres con las mujeres de los distintos municipios para identificar los daños ocasionados y la violación de los derechos individuales y colectivos con sus respectivos impactos. Este proceso de planeación para la construcción del diagnóstico del daño por parte de la OFP y el seguimiento de su comité de impulso o de víctimas ha permitido generar estrategias de acompañamiento al proceso.

\section{Avances de los comités de impulso frente al proceso de la reparación colectiva}

El proceso de reparación colectiva cuenta con una serie de pasos que inicia desde el reconocimiento de los sujetos colectivos, el diagnóstico del daño, la construcción de medidas hasta su misma implementación y seguimiento. En la actualidad los sujetos de reparación colectiva de Ciénaga del Opón y la Organización Femenina Popular se encuentran en etapa de implementación de medidas (30 medidas de Ciénaga del Opón y 41 medidas de la OFP).

La construcción de las medidas de reparación. En la etapa de diagnóstico del daño, que es trabajada en conjunto por los sujetos de reparación colectiva y la Unidad de Víctimas, se recogen las narraciones, hechos, informes y evidencias que establezcan los posibles daños colectivos ocasionados en el marco del conflicto y, con este resultado, se estipulan las medidas de reparación en un documento denominado Plan Integral de Reparación Colectiva (PIRC). Desde finales del 2012 y comienzos del 2013 la Unidad de Víctimas convoca a la comunidad de Ciénaga del Opón y la OFP con el fin de adelantar el proceso de reparación, ambos sujetos aceptan participar, no sin antes generarse una serie de discusiones internas frente a la convocatoria del Estado. 
El debate interno en la OFP estaba atravesado por la necesidad de iniciar un proceso de reparación que incluyera el restablecimiento de la confianza hacia un Estado que, en muchos casos, ya sea por acción u omisión, ocasionó parte de sus hechos victimizantes. Otro elemento de resaltar en esta parte del proceso, es que la realización del diagnóstico del daño fue hecho por las mismas integrantes de la organización, respaldadas por su proceso de formación y empoderamiento como sujetas políticas, junto a la asesoría brindada por distintos organismos nacionales e internacionales.

Para la comunidad de Ciénaga del Opón el acercamiento con la Unidad de Víctimas para el desarrollo del diagnóstico del daño y la construcción de medidas se hace bajo la percepción de la institución de que la población víctima a reparar se encontraba concentrada en la cabecera del corregimiento; es allí donde los distintos líderes comunitarios presentes hacen manifiesto la necesidad de vincular no solo a la cabecera, sino a las demás veredas del corregimiento (cuenta con nueve veredas: Cabecera, Ñeques, Florida, Colorada, Candelaria, Caño rasquiña, Playón, Santo Domingo y Comes y Llevas), pero al final solo cuatro fueron reconocidas por parte de la Unidad de Víctimas como sujeto de reparación y este argumento de la institución se fundamenta en que solo cuatro veredas sufrieron el total desplazamiento de su población allí asentada (Cabecera, Ñeques, Florida y Colorada), trayendo inconformismo de la comunidad con la Unidad de Víctimas.

En vista de las diferencias dadas en esta fase del proceso de reparación junto al desconocimiento y poca experiencia en lo referente a los procesos a desarrollar, la comunidad de Ciénaga del Opón con su respectivo comité de impulso deciden buscar acompañamiento del Programa de Desarrollo y Paz de Magdalena Medio, con quienes ya habían tenido acciones conjuntas en el contexto del retorno de los pobladores al corregimiento.

Finalmente, y después de muchas negociaciones, se aprueban en el 2015 los planes integrales de reparación de ambos sujetos colectivos, quedando estipulado para Ciénaga del Opón 30 medidas que se orientan hacia la reparación material (mejoramientos de escuelas, puesto de salud, puente de ingreso al corregimiento, cuidado de la ciénaga, etc.), reparación simbólica (día del retorno, fiestas patronales, etc.), al igual que estrategias de ayuda psicosocial y de formación (escuela para jóvenes y formación según la vocación del corregimiento). Y para el caso de la OFP se estipularon 41 medidas que también incluyen la reparación material (casa de encuentro de las mujeres, dotación, planes de viviendas, etc.), la reparación simbólica (institucionalización del día de la mujer) y la reparación política y psicosocial (política pública de mujer y equidad de género, escuelas de formación para mujeres y espacios culturales juveniles, entre otros).

Implementación y seguimiento de medidas. Dentro de lo estipulado por parte de la Unidad de Víctimas, el proceso de construcción del PIRC consta de un periodo de seis meses 
y la implementación tendrá un tiempo de tres años para su total ejecución. Es decir, que si el PIRC de ambos sujetos está aprobado desde el 2015, ya para el año 2018 se tendrían que implementar en su totalidad las medidas de reparación. Sin embargo, la realidad ha sido otra. La construcción del PIRC abarcó un periodo de dos años y a fecha actual, y como se mencionó al comienzo de este capítulo, para el caso de Ciénaga del Opón solo se han implementado las medidas de tipo simbólico y psicosocial: la conmemoración del día del retorno, la estrategia de recuperación del tejido social y la institucionalización de las fiestas patronales del corregimiento. Pero las medidas restantes, concernientes a infraestructura, implementación de proyectos como mantenimiento y conservación de las fuentes hídricas, dotación de escuelas y puestos de salud, entre otras medidas, están sin ejecutar por parte de los entes competentes.

Para el caso de la OFP, se han implementado nueve medidas de forma completa y nueve medidas parciales, entre estas últimas se encuentra el mejoramiento de las Casas de la Mujer, los espacios culturales, las escuelas para los jóvenes, que representan un 58\% de avance del proceso.

La ejecución de las medidas de los PIRC es asumida por las administraciones municipales y se enlazan con los planes de acción territorial (PAT) de los respectivos municipios donde se asigna el recurso financiero requerido para ser ejecutados. Parte del incumplimiento en la ejecución de las medidas se debe, entre otros factores, a:

1) El desconocimiento de las administraciones en distinguir las políticas sociales para las diversas poblaciones, del enfoque diferencial que se debe tener para los sujetos de reparación colectiva; por ejemplo, dentro de los planes de desarrollo municipales se estipulan subsidios de vivienda para la población víctima, mientras que en los PAT se estipula la adecuación de las casas para la mujeres de la OFP o los habitantes de Ciénaga del Opón; esto conlleva a que las inversiones se hagan de forma general (inversión a todo tipo de vivienda en el municipio), sin tener en cuenta el origen de la medida (hecho victimizante), las particularidades y características del sujeto de reparación y la prioridad que requiere la población víctima.

2) Desde las secretarias de despacho se desconocen las medidas del PAT por lo cual no centran esfuerzos en su cumplimiento.

3) Desde el nivel nacional hasta municipal se ha desbordado la capacidad institucional para atender a las víctimas, por lo cual los recursos no alcanzan para todos los procesos de reparación.

Los comités de impulso han emprendido acciones de seguimiento como acudir a los entes de control como Procuraduría y Defensoría del Pueblo, realizar intervenciones ante los consejos municipales y radicar derechos de petición. Los comités de impulso se encuentran en un proceso de acudir a las distintas instancias que permite la ley y la constitución para garantizar 
el cumplimiento de dichas medidas, aunque este proceso no garantiza el cumplimiento inmediato, si permite fortalecer las dinámicas grupales y una mayor incidencia política.

\section{Conclusión}

\section{Consideraciones finales}

En el marco de la investigación realizada se evidenció que las dinámicas grupales y procesos organizativos se definen según la fase grupal. En este sentido, el comité de impulso de Ciénaga del Opón se encuentra en transición de una fase formativa hacia una fase intermedia, en donde la primera se caracteriza por la identificación de intereses comunes con el fin de establecer parámetros de comportamiento, y en la intermedia, se encontrarán con relaciones interpersonales consolidadas, niveles moderados de cohesión y nuevos roles o perfiles internos (Gnecco, 2005). Esto debido a que sus dinámicas grupales se desarrollan de manera informal y orientada a la operatividad, lo que puede ofrecer algunas ventajas como la agilidad al momento de decidir, comunicar o establecer criterios frente al proceso, pero mostrando debilidad a la hora de abordar los momentos álgidos y complejos de las distintas etapas de la reparación colectiva.

En cuanto al comité de la OFP, se puede afirmar que este se encuentra en una fase de madurez grupal, cuya característica principal es la permanencia en el tiempo y la consolidación de esquemas y políticas de comportamiento (Gnecco, 2005). De ese modo, se establece una considerable ventaja en materia de dinámicas grupales y a su vez en materia de resultados por parte de los comités que representan a las organizaciones sociales, ya que sus procesos de liderazgo, distribución de roles, comunicación, cohesión y planeación, se han formado previamente al proceso de reparación en procesos organizativos que han conllevado décadas. Por su parte, los comités de base comunitaria se crean para responder a un asunto que desconocen en su funcionamiento, aunque no en su importancia; en esa medida, son nuevos y están en el proceso de reconocerse como grupo y consolidar su dinámica.

Para los comités de impulso de base organizativa como la OFP, el proceso de reparación colectiva representa una estrategia dentro de su organización, lo cual no determina su continuidad como grupo, mientras que para los comités de base comunitaria representa su único objetivo que de cumplirse llevará a estos comités a replantear su acción futura, ya sea terminando el comité o replanteando su accionar desde otro espacio o figura organizativa. La experiencia previa de las organizaciones sociales y el apoyo que le brindan a su comité de impulso posibilitan mayor incidencia política frente al proceso de reparación. En esa medida, la intervención del trabajo social en comités de impulso de base comunitaria se convierten en un desafío profesional en tanto representan a comunidades que fueron fragmentadas por la guerra y hoy deben volverse a organizar para exigir sus derechos ante el Estado. 
En ese sentido, la intervención del trabajo social con comités de impulso de reparación colectiva debe priorizar aquellos que son de base comunitaria, pues son los que cuentan con menos herramientas para enfrentar el proceso de reparación con éxito. Las acciones profesionales deben estar orientadas a fortalecer los procesos de liderazgo, mejorar la comunicación tanto dentro del comité de impulso como hacia la comunidad que representa, propiciar escenarios para la planeación de las acciones del comité e implementar estrategias que mejoren la cohesión del grupo, entre otros aspectos.

En cuanto a los resultados sobre las acciones que implementan los comités de impulso para lograr el cumplimiento de las medidas de reparación, se concluye que independientemente de la dinámica grupal que caracterice al comité, el cumplimiento de las medidas es directamente proporcional a la voluntad política de las instituciones encargadas de ejecutar el plan de reparación, al igual que la disponibilidad de recursos económicos por parte de dichos entes. Además, otra situación que dificulta la implementación de las medidas de reparación es que el porcentaje de víctimas en Colombia (14\% de la población) es mayor que el de otros procesos de reparación en el mundo, por ejemplo, en Perú, Guatemala, Indochina y Sudáfrica la población víctima fue alrededor del 1\% de la población del país (Sikkink, Marchesi, Dixon y D’Alessandra, 2014), desbordándose así la capacidad institucional del Sistema Nacional de Reparación de Víctimas, SNARIV. También, debe considerarse que no existe una distinción clara entre los programas que deben reparar los daños causados en el conflicto y las necesidades básicas generales de la población.

A pesar de este dificultad de carácter estructural que sobrepasa las responsabilidades de los comités de impulso se puede decir, en concordancia con el proyecto ético-político de trabajo social, que el fortalecimiento de estos colectivos puede considerarse como una estrategia profesional para el fortalecimiento de los procesos comunitarios y de la capacidad de las comunidades para interlocutar con las entidades estatales en la exigibilidad de sus derechos.

\section{Referencias}

Ander Egg, E. (2003). Introducción al trabajo social. Buenos Aires, Argentina: Lumen.

Ander Egg, E. (2007). Introducción a la planificación estratégica. Buenos Aires, Argentina: Editorial Lumen.

Bass, B.M. \& Riggio, R.E. (2006). Transformational Leadership. New York: Psychology Press.

Congreso de la República. (10 de Junio de 2011). Ley 1448. Por la cual se dictan medidas de atención, asistencia y reparación integral a las víctimas del conflicto armado interno y se dictan otras disposiciones. Diario Oficial. 
Fernández, C., Cancino, D., Botero, L.F. y Prada, M.P. (2016). Facilitación de diálogos y transformación de conflictos conceptos y herramientas básicas para la práctica. Bogotá, Colombia: Fundación RedprodePaz.

Festinger, L., Schachter, S. y Back, K. (1950). La presión social de los grupos informales. Un estudio de los factores humanos en la vivienda. Nueva York: Harper y Row.

Galtung, J. (1999). Tras la violencia, 3r: reconstrucción, reconciliación, resolución:Afrontando los efectos visibles e invisibles de la guerra y la violencia. Bilbao, Manizales: Bakeaz.

Gnecco, M.T. (2005). Trabajo social con grupos, fundamentos y tendencias. Bogotá, Colombia: Kimpres.

Hogg, M.A. (1992). La psicología social de la cohesión grupal desde la atracción a la identidad social. Londres: Harvester Wheatsheaf.

Huici, C. (2012). Composición y estructura del grupo. En C. Huici., F. Molero., Á. G. Gómez. y F. Morales. (Eds), PsicologÍa de los grupos (pp. 125-172). Madrid, España: Universidad Nacional de Educación a Distancia.

Knowles, M.S. y Knowles, H. (1962). Introducción a la dinámica de grupo. Ciudad de México, México: Letras.

Lederach, J.P. (1989). Elementos para la resolución de conflictos. Ciudad de México, México: Publicación del Servicio Paz y Justicia.

Macías, G. (2003). Teorías de la comunicación grupal en la toma de decisiones:contexto y caracterizaciones (tesis de doctorado). Universidad Autónoma de Barcelona, Barcelona, España.

Mesa Nacional Coordinación Sujetos de Reparación Colectiva. (2018). En Colombia más de 600 casos de reparación colectiva no han sido concluidos. Recuperado de https://www.arcoiris. com.co/2018/05/en-colombia-mas-de-600-casos-de-reparacion-colectiva-no-han-sidoconcluidos/.

Morelo, F. (2012). El Liderazgo. En C. Huici., F. Molero., Á.G. Gómez. y F. Morales. (Eds), PsIcología de los grupos (pp. 173-210). Madrid, España: Universidad Nacional de Educación a Distancia.

Obando, D.E. (2016). Vidas de historia, Memoria literaria de la OFP. Barrancabermeja, Colombia: Organización Femenina Popular.

OFP. (Sin Fecha). Organización Femenina Polular. Recuperado de http: / / organizacionfemeninapopular. blogspot.com/p/inicio.html.

PDPMM. (2012). Espacio Humanitario de Ciénaga del Opón recolección de memoria histórica de sus pobladores. Barrancabermeja, Colombia: [s.n]. 
Roda, R. (1999). La estructura del grupo: estatus, rol, normas y cohesión. En G. Francisco. y C. Alcover de la Hera. (Eds), Introducción a la Psicología de los Grupos. Madrid, España: Ediciones Pirámide.

Rossell Poch, T. (1998). Trabajo social de grupo: grupos socioterapéuticos y socioeducativos. Cuadernos de Trabajo Social, (11), 103-122.

Schafers, B. (1984). Introducción a la sociología de grupos: historia, teorías, análisis. Barcelona, España: Herder.

Shaw, M. (1983). Dinámica de Grupo. Psicología de la Conducta de los Pequeños Grupos. Barcelona, España: Herder.

Sikkink, K., Marchesi, B., Dixon, P. y D’Alessandra, F. (2014). Reparaciones Integrales en Colombia:Logros y Desafíos Evaluación Comparativa y Global. Boston: Harvard Kennedy School Carr Center for Human Rights Policy.

Yukl, G. (2008). Liderazgo en las organizaciones. Madrid, España: Pearson Educación. 